\title{
Sobre el término medieval cocatriz, variantes y acepciones
}

\author{
Alida Ares Ares \\ Universidad de Trieste
}

De entre los nombres de animales que en su forma vulgar suscitan dudas centramos nuestra atención en la voz cocatriz, que nos ha interesado tanto en sí misma como por su posible uso traslaticio en el Libro de buen amor $(L b a)$, limitando nuestro análisis al ámbito francés e hispánico y, en especial, a aquellos casos en los que la voz está denominando al cocodrilo o a animales que en la tradición literaria se encuentran en estrecha relación con éste'.

En el verso 881c del Lba: "Castigad vos amiga de otra tal contra yz" (ms. Salamanca) / "castigat uos amiga de otra tal contratriz" (ms. Gayoso), la enmienda cocatriz que proponía por primera vez Joan Corominas en su edición (JUAN RUIZ, 1967), ha sido muy controvertida, incluso desde el punto de vista del significado de la voz. Sin embargo consideramos que la interpretación cocatriz: 'cocodrilo', que Jacques Joset definía "muy sospechosa" y G. Gybbon-Monypenny "extraña y pura fantasía"2, es digna de consideración ${ }^{3}$.

Anticipamos la relación que existe entre la voz cocatriz, referida al crocodilus niloticus, y la misma referida indirectamente, al (herpestes) ichneumon: 'mangosta', también llamado por otro nombre en(h)ydrus, y vulgarmente murem Pharaonis, animal considerado por los antiguos como uno de los enemigos mortales del cocodrilo, y el cual es confundido frecuentemente, desde la Antigüedad y durante la Edad Media, con otros mamíferos carniceros morfológicamente semejantes, como la nutria y el hyllos o suillo (animal no bien identificado) ${ }^{4}$.

1 Un estudio sobre la voz cocatriz en la literatura inglesa, lo ha llevado a cabo P.A. RoBIN (1932), 83-95; y un breve resumen del mismo T.H. WHITE en The Book of Beasts (1954), 169-170.

2 Juan Ruiz (1974), 308, donde Jacques Joset en nota: "Frente a la dificultad de interpretación, Corominas cambia contraíz (S) / contratriz (G) en un cocatriz: 'cocodrilo' muy sospechoso". JUAN RUIz (1988), 290, Gybbon-Monypenny en nota: "La enmienda cocatriz de Corominas (que él traduce extrañamente por "cocodrilo" -ino sería el animal heráldico que en inglés se llama cockatrice?), es pura fantasía".

3 Los versos ya han sido objeto de una disquisición de JOSÉ JURADO (1988).

4 Para lo que se refiere a la zoografía, véase Bernhard Grzimek (1972). Acerca de los herpestes vol. 12, 187 y 194; para la familia de las lutrinae, vol. 12, 90-105. 
La ignorancia acerca de animales exóticos para el país y la similitud fonética del nombre, provocaron confusión, ya desde la Antigüedad, entre en(h)ydrus referido al ichneumon, en(h)ydris 'nutria' e hydrus 'serpiente de agua' (todavía hoy en la mayor parte de los diccionarios no se establece una diferencia clara, ni entre ésta última e hydra, la serpiente mitológica). En algunos textos medievales encontramos la voz cocatriz referida a hydrus (var. ydre), término que había reemplazado en ciertos episodios a ichneumon.

Por otra parte, sea por corrupción fonética de la voz crocodilus en lengua vulgar, sea por la ambigüedad de algunos pasajes manuscritos, se identificaron también cocatriz y cocodrilo.

Hemos intentado reunir aquí algunos textos que permiten rastrear la historia de esta relación terminológica, partiendo de los tratados sobre animales de los autores clásicos, y en particular del capítulo en el que tratan del cocodrilo.

Plinio (23-79 d. de C.), en Naturalis Historia, describe la lucha entre el ichneumon y el cocodrilo. El capítulo fue parafraseado después por muchos autores que introdujeron modificaciones léxicas:

Hunc [crocodilum] saturum cibo piscium et semper esculento ore in litore somno datum parva avis, quae trochilos ibi vocatur, rex avium in Italia, invitat ad hiandum pabuli sui gratia, os primun eius adsultim repurgans, mox dentes et intus fauces quoque ad hanc scabendi dulcedinem quam maxime hiantes, in qua voluptate somno pressum conspicatus ichneumon, per easdem fauces ut telum aliquod inmissus, erodit alvum (PLINIUS, 1986, I. VIII, cap. 37).

Algunos particulares que refiere Plinio en este pasaje, ya documentados en Herodoto y en Aristóteles, han sido confirmados por los naturalistas modernos, como el hecho de que los cocodrilos permanezcan durante horas inmóviles en la orilla del río con las fauces abiertas y que algunos pájaros osen introducirse en su boca para extraer sanguijuelas adheridas, e incluso pececillos (GrZIMEK, 1972, vol. 6, 152-153). No ha sido demostrado en cambio hasta ahora que la mangosta (ichneumon) u otro animal se introduzca por su garganta y lo mate. Sin embargo, con independencia de la veracidad del relato, nos interesa en particular la terminología empleada para denominar al animal en la primera parte de nuestro análisis.

Solino (s. III) en Collectanea Rerum Memorabilium (Solinus, 1864, cap. 35), en un episodio similar al de Plinio, llama al ichneumon, enidro, especificando que se trata de "alterum ichneumonum genus". La glosa es recogida por Ammiano Marcelo (s. IV-V) en Rerum gestarum: "enhydrus in Syria ichneumonis genus" (AMmiano MARCELO, 1963, XII, 15, 19). 
C. Salmasio en su comentario a la obra de Solino, Exercitationes in Solinum Plinianas (SALMASIO, 1689, 316-317), postula que el hecho de que Solino llame enidros al ichneumon, considerándolo un animal acuático, proviene de un pasaje de Estrabón que describe cómo el ichneumon, agarrando por la cola al áspid, lo arrastra hasta el río y lo mata (STrabo, 1852, vol. 3, I. XVII). También Vitruvio incluye entre los animales del Nilo al ichneumon, junto con los cocodrilos y los hipopótamos, como si se tratara de un animal acuático, o bien anfibio ${ }^{5}$. En cambio el mismo Estrabón, más adelante, lo clasificó entre los animales terrestres, al igual que Aristóteles (ARISTOTELES, 1927, I.VI, cap. 35) Plinio (PLINIUS, 1986, I. VIII, cap. 36) y otros muchos autores.

Sin duda el ichneumon vive entre las arenas, a orillas del Nilo, cerca del agua, pero no en ella. Enidros (del gr. $\varepsilon v v \delta \rho o s$ 'el que vive en el agua') sería -según Salmasio- lo que en latín se llama lutra, 'nutria' (gr. $\varepsilon v v \delta \rho i ́ s)$, animal muy abundante en el Nilo. Solino, por error, habría hecho del ichneumon un género de enhydrus, a pesar de ser éste un animal distinto. No obstante, como veremos, enidros, interpretado como sinónimo de ichneumon, gozó de gran fortuna durante la Edad Media, sobre todo gracias a su transmisión en los bestiarios.

Timotheo de Gaza (s. V-VI), en De animalibus, agrega otro término, hyllos, como sinónimo: "ichneumon, el cual se llama también sea hyllos, sea enhydros" (HAUPT, 1869, caps. 42 y 43). Y Georgios Pisides (s. VII) en Hexaemeron (PISIDES, 1860, 1507 y ss.) emplea asimismo hyllos para denominar al ichneumon. En la ya citada obra de Salmasio, comentando este pasaje de

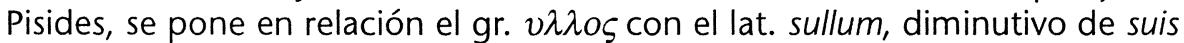
'puerco' ${ }^{\prime}$, nombre que, según el autor, se le habría asignado al ichneumon por la similitud de su rostro con el de los suilli o "quod ut sues semper rostro quaerat et investiget", aludiendo a la etimología de ichneumon (gr. $\imath \chi v \varepsilon v \mu \omega v)$ que significa "el que sigue las huellas, busca o investiga".

La voz suillus, en relación con enhydros e ichneumon, se encuentra en Etimologías de S. Isidoro, donde se cita un pasaje de De Laudibus Dei de Draconcio:

36. Enhydros bestiola ex eo nuncupata, quod in aquis versetur, et maxime in Nilo. Quae si invenerit dormientem corcodilum, volutat se in luto primun, et intrat per os eius in ventrem, et carpens omnia intranea eius, sic moritur. 37. Ichneumon Graece vocatus, eo quod odore suo et salubria ciborum et venenosa produntur. De quo Dracontius ait (Laud. 1,515): "Praecidid suillus vim cuiuscumque veneni" (S. ISIDORO, 1911, I. XII, 2, 36, 37).

5 VITRUVIO, De architectura, I. VIII, cap. 2 (apud SALMASIO, 1689, 316).

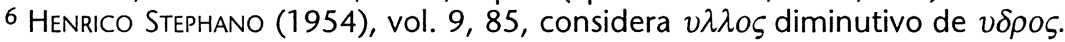


Observamos que se encuentran en parágrafos diversos ichneumon y enhydros. En cambio, como hemos visto, Solino y A. Marcelo los identifican; por lo que proponemos interpretar las palabras de Isidoro como referidas a un solo animal. También el THeSAURus LINGUAE LATINAE (1900, t. VII, s.v. ichneumon), remitiendo a este pasaje de Isidoro, conviene en que allí los tres términos, ichneumon, enhydros y suillus, son sinónimos.

Hay que precisar, sin embargo, que según las ediciones que han llegado hasta nosotros, en Laudibus Dei I, 515, no aparece el término suillus, sino Psyllus, referido a un pueblo de Libia que encantaba serpientes y sabía curar sus mordeduras. La variante suillus se encuentra en la recensión del texto de Eugenio Toletanus (Blosius Aemilius Dracontius, 1961).

La hipótesis que sostienen algunos críticos, como J. Oroz Reta y M. A. Marcos Casquero, es que se trata de una interpretación errada de Isidoro del texto de Draconcio (S. ISIDORO, 1982, vol. II, 76, en n. 37). Por otra parte, en Etimologías XII, 6, 12 encontramos de nuevo suillus pero aquí referido a un tipo de pez, el sollo: "Porci marini, qui vulgo vocantur suilli"?.

La relación del "suillo" con el ichneumon podría limitarse sólo a rasgos morfológicos ${ }^{8}$, sin embargo, convendría profundizar en el texto de Isidoro, ya que puede provenir de hyllos, como sostiene Salmasio, y además existen "suillos" que se asemejan al ichneumon, como el suillotaxus marchei, una especie de tejón que tiene el hocico parecido al del puerco, e incluso el hilomys suillus, de la familia de los gimnuros?.

En la Edad Media, en las secciones de historia natural de las obras enciclopédicas, abundan las descripciones de animales como objeto de disquisición. En el fondo, como es sabido, todas tratan de un mismo material y los autores se copiaban unos a otros sin acudir a la observación de la realidad. Dos de ellas, Li livres dou Tresor de Brunetto Latini y la General Estoria, de Alfonso $X$, nos interesarán especialmente para el estudio de la voz cocatriz (vid. infra). Junto a éstas encontramos los bestiarios, en los que a la descripción de animales, sin preocupaciones científicas, se acompaña una interpretación simbólica moralizadora. Estos últimos proceden del Physiologus griego (s. II) del que hubo abundancia de manuscritos y traducciones hasta el s. XIII.

7 Esta definición de suillo aparece recogida en ALONSO DE PALENCIA (1967), t. II, s.v. suillus. Y en H. DE S. VICTOR (1879), De Bestiis et aliis Rebus, I. III, cap. 55. De paso advertimos que la interpretación suillus: 'sollo', es rebatida por E. DE SAINT-DENIS (1947), 90 y 111, donde comenta el texto de Isidoro.

8 Cfr. A. FORCELLINI (1940), t. II, 697, s.v. ichneumon: "animal est magnitudine felis, specie muris, vulgo mus Indicus, quod nascitur in Aegypto, et cum aspide pugnat: ab igneio, investigo, sive quod instar canis venatici investiget crocodilum, et aspidem, ovaque eorum observet, sive potius quod rostro suo, quod est simile rostro suillo, cibum ruspetur et investiget".

9 Véase Grzimek (1972), vol. 12, 81-83 y vol. 10, 203-204, respectivamente. 
El texto del Physiologus ha sufrido muchas modificaciones, en particular, en lo que se refiere al orden de los capítulos. En la edición moderna de F. Sbordone, cap. 25, se denomina enidros al enemigo del cocodrilo (Physiologus, 1936, 87-88). El ichneumon aparece en el cap. 26 como el enemigo de las serpientes; pero ya algunos críticos, como Otto Seel, no excluyen que se trate de un "doblete"10. Por influencia del Physiologus, encontramos en los bestiarios de los siglos XIII-XIV las voces ydrus, ydre, ysdre, derivados o corrompidos de enidros, para denominar al enemigo del cocodrilo.

Hemos de advertir que, paralelamente a la transformación terminológica, se va produciendo también una especie de "metamorfosis" del animal, de lo que dan fe los dibujos y grabados que ilustran a menudo muchos de los tropos. En las obras clásicas más antiguas ichneumon, así como en(h)ydrus (ichneumon), se puede identificar con lo que hoy conocemos como la mangosta. Muy interesantes a este propósito son los emblemas que figuran al trochilo dentro de la boca del cocodrilo y su lucha con el ichneumon"1.

En Physiologus se dice de enidros que tiene la forma de un perro. En la versión italiana de F. Zambon ${ }^{12}$ y en la alemana de Otto Seel se traduce enidros por lontra y Fischotter, respectivamente, 'nutria'13.

En el primer bestiario en lengua vulgar de Philippe de Taün (s. XII), encontramos ya ydrus identificado con culovre, no obstante la crítica lo presente como una traducción en verso del Physiologus (TAün, 1970, vv. 663 y ss.). En Le Bestiaire divin de Guillaume le Clerc (s. XIII), ydrus es también "une maniere de serpent" (CLERC, 1970, cap. 19, 244-245); posteriormente, en Le Bestiaire d'Amour de Richard de Fournival (s. XIII) y en La Réponse de la Dame (s. XIV) la ydre y la ysdre, respectivamente, se identifican con la hidra mitológica $^{14}$. Y es así también como se describe en los bestiarios catalanes recogidos por S. PANUNZIO: "E és una altra natura de serpent, qui-ha molts caps, e com hom li n'ha tallat un, nexen-li'n dos"15.

10 Physiolocus (1960). En n. 111: "Wahrscheinlich ist die Pharaonsratte gemeint. Der in Nr. 26 folgende Ichneumon ist wohl einfach eine Dublette: die Panzerung mit Lehm und die Feindschaft gegen das Krokodil wird in der Antike oft erwähnt (Arist., Str., Ael., Plut., Plin., Opp., etc.)".

11 Véase A. Henkel y A. SChÖne (1967), 667-676, y cfr. Joaquimo Camerario (1654), 97-101.

12 PhYSIOlocus (1975), cap. 25, 63: "Esiste un animale detto lontra, che ha la forma de un cane, ed è nemico del coccodrillo".

13 Cfr. CovarRubias (1953), s.v. nutria: "Quasi lutria, latine lutra, enhydrus".

14 Fournival (1978). La Rèponse se creyó en un principio obra del mismo FourNIVAL, pero hoy se sabe que pertenece a una época posterior (s. XIV). Véanse las ilustraciones relativas a la ydre y al cocodrilo en las páginas 35 y 88 .

15 Saverio Panunzio (1988), vol. I, cap. 26, 101-104. Se transcribe el ms. 75 (s. XV) de la Bibl. Univ. de Barcelona, con variantes del ms. 310 (s. XV) de la Bibl. de Cataluña. 
Las fases que hemos seguido hasta aquí pueden resumirse en un proceso que llevaría de ichneumon 'mangosta' a enidros 'un género de ichneumon o bien nutria', según los textos, y de éste a ydrus / ydre / ydra 'serpiente de agua o hidra'.

Pasemos a analizar a continuación el término cocatriz y cómo se ha producido la identificación con ydre y con cocodrilo.

Un libro muy difundido en la Edad Media y que creemos que ha contribuido a la confusión de las tres voces es Li livres dou Tresor (Tresor) de B. Latini. De él existen dos ediciones modernas, una de P. Chabaille ${ }^{16}$, de 1863 y otra de F. J. Carmody ${ }^{17}$, de 1948 . El capítulo dedicado al cocodrilo es muy confuso. Las dos ediciones presentan variantes respecto a la interpretacion de la voz cocatriz.

Hay que tener en cuenta que del original brunettiano fueron hechas dos redacciones en un breve arco de tiempo, una en Francia entre 1260 y 1267 , y otra poco después del regreso de Latini a Florencia en 1267, y hasta el momento no ha sido llevada a cabo una recensio codicis que tenga en cuenta las divergencias fundamentales de ambas y los recientes descubrimientos $^{18}$.

Citamos en primer lugar el pasaje donde se alude a la cocatriz de la edición de Chabaille:

\section{DOU COCODRILLE ET DOU COCATRIS.}

Or avient que quant li oisiaus qui a non strophilos vuet avoir charoigne por mangier il se boute en la bouche dou cocodrille, et li grate toute belement, tant que il oevre toute sa gorge pour le grant delit dou grater. Lors vient $\mathrm{i}$ autres poissons qui a nom ydre, ce est cocatris, et li entre dedanz le cors, et s'en ist de l'autre part, brisant et derompant son oste, en tel maniere que il l'ocist.

Neis li dalphin meisme, qui ont aussi come une sie sor le dos, quant il le voient noer, il s'en entrent desouz et le fierent emmi le ventre si que le font devier maintenant.

16 B. LATINI (1863). Basada en el ms. 198 de la Biblioteca Imperial, fechado en 1284 (en vida del autor). Ms. fr. 12581 de la Bibliothèque Nationale de Paris.

17 B. LATINI (1948). Basada en el ms. 1110, proveniente de la Biblioteca de Gian Galeazzo Visconti, que se encuentra en la Bibliothèque Nationale de París.

18 Véase MARCELLO CiCCUTO (1992), 45-57. Se refiere en particular a los estudios de JULIA B. HollowaY (1986), que agrega otros manuscritos descubiertos recientemente a la lista de cerca de ochenta franceses proporcionada por CARMODY en su edición. 
Et sachiez que cocatriz, ja soit ce que il naist en l'aigue, et vit dedans le Nile, il n'est mie peisson, ainz est serpens d'aigue; car il ocist l'ome que il peut ferir, se fiens de buef ne le garit. (LATINI, 1863, I, 5, 132, 184-185; la cursiva es nuestra)

Las tres palabras, "ce est cocatris", referidas a ydre, no aparecían en el ms. 198. Chabaille las añadió en el texto basándose en otros manuscritos: ms. 20 de la Biblioteca del Arsenal, mss. 7066 y 7930 de la Biblioteca Imperial, y ms. 697 de la Biblioteca de la villa de Lyon. En nota recoge además otra variante: "Idre, ou volés vos qualquetrix, qui toute est une chose", que se encuentra en los mss. 7067, 7160 y 7366 de la Biblioteca Imperial. El título apoya la tesis de que el cocodrilo y la cocatriz son dos animales distintos.

En la vulgarización del texto al italiano del s. XIII, atribuida a B. Giamboni $^{19}$, ya no se menciona ydre, que queda sustituida por calcatrice:

\section{DEL COCCODRILLO.}

Or avviene che quando uno uccello chiamato sconfilions vuole carogna, va a questo animale, e ponesi alla boca, e grattali la gola si dolcemente ch'egli apre la bocca. Allora viene un altro animale ch'ha nome calcatrice ed entrali dentro lo corpo [...] II simigliante fa il delfino [...] E sappiate che la calcatrice, con tutto ch'ella nasca in acqua e viva nel Nilo, ella non è pesce, anzi è serpente d'aqua, che ella uccide l'uomo se'l puote ferire, se fegato di bue non lo guarisce.

Luigi Carrer, autor de la edición, advierte la confusión terminológica del texto al compararlo con las fuentes del Tresor: las obras citadas de Plinio y Solino, donde la calcatrice, como ya hemos visto, es llamada ichneumon y enidro, respectivamente.

A estas fuentes proponemos añadir Etim. XII, 4, 22, que podría haber actuado como filtro, como hace sospechar el último párrafo. En efecto, allí donde se trata de la hydros, la serpiente de agua, Isidoro dice: "Hydros aquatilis serpens, a quo icti obturgescunt; cuius quidam morbum boam dicunt, eo quod fimo bovis remedietur". Este dato del modo de curar la mordedura de la hydros, se recoge en el texto de Latini, que traduce fimo por fiens 'estiér$\mathrm{col}^{\prime}$, mientras que Giamboni erróneamente lo traduce por fegato (esp. 'higado'). Es probable que Latini haya confundido la hydros con enhydros de Etim. XII, 2, 36, el enemigo del cocodrilo ${ }^{20}$. Esto explicaría la corrección dentro del

19 B. LATINI (1839), 199-200. En italiano existen unos 30 manuscritos del Tesoro. La edición más moderna es la de Luigi Gaiter (B. LATINI, 1871). 
mismo capítulo: " $n$ 'est mie peisson, ainz est serpens d'aigue [...]". Giamboni esquiva la contradicción del texto traduciendo "autres poissons" por "un altro animale". El capítulo es un buen ejemplo de las dificultades de los copistas y de los traductores (vid. infra los textos catalán y castellano).

En España el libro gozó de una gran popularidad. En la actualidad se conocen 14 manuscritos en lengua castellana ${ }^{21}, 4$ en catalán y 1 en aragonés. Existen dos ediciones modernas: la de Spurgeon Baldwin ${ }^{22}$, de la traducción castellana, basada en el ms. 685 de la Biblioteca Nacional de Madrid, del s. XV, con enmiendas de otros mss., y la de C. J. Wittlin, de la traducción catalana, basada en la única versión completa conservada, la de Guillem de Copons, de 1418, que corresponde al ms. 357 de la Biblioteca de Cataluña (LATINI, 1976).

El texto catalán coincide con la edición de Chabaille en que cocatriz se identifica con idre. Así en el vol. II, cap. 125, Copons traduce la variante recogida por Chabaille: "Idre, ou volés vos qualquetrix, qui toute est une chose":

Del Corcorell, qui ha IIII peus

E sdevé-sse que quant un ocell qui ha nom strafillos volhaver caronya per menjar, ell se met en la bocha del corcorell, el grata tan suau tro que ell obra la bocha, per le delit del gratar; lavors ve un peix qui ha nom idre, 0 , si volets, calcatrix, que és tot hu, e li entre dins lo cors, e se'n hix d'altre part [...] Mas los dalfins mateixos [...] E sapiats que calcatrix nasquen dins lo riu del Nil e dins aquell viu; no és peix ans és serp de vida; e ociu home si.l fér, si no.l guareix fem de bou.

Veamos a continuación los textos donde cocatriz se identifica con cocodrilo, partiendo de los bestiarios y otros textos franceses, el mismo Tresor de Latini (ed. Carmody) y la traducción de éste al castellano.

Los bestiarios franceses del XIII, Le bestiaire divin, de Guillaume le Clerc (h. 1210), Le bestiaire d'amour de Fournival (h. 1250) así como su complementario, Le Rèponse della dame (s. XIV), documentan cocatriz, con las varian-

20 Un testimonio más próximo a LATINI donde también se produce la identificación de los dos animales lo ofrece HuGo DE S. VICTOR (s. XII), De Bestiis, I. II, cap. VII: "Inde hydrus aquaticus serpens, cujus ictu obturgescunt, quem morbum boam dicunt, eo quod fimo bovis curetur [...] At hic hydrus praefatus inimicus est crocodilo, et hanc habet naturam et consuetudinem, ut cum venerit et viderit crocodilum in littore dormientem, vadit et involuit se luto, quo possit facilius illabi in fauces crocodili".

21 Además de los 13 que eran conocidos, J. B. Holloway ha descubierto recientemente otro en la Biblioteca Colombina de Sevilla, cód. 5-I-6, del que da noticia MARCELLO CICCUTO (1992), 45.

22 B. LATINI (1989). Existe también una edición anterior del mismo Baldwin (LATINI, 1982). 
tes coquatriz, caucatrix y chocatrix, respectivamente, atribuyéndole el significado de cocodrilo. A saber:

Le coquatriz est beste fiere

Et vit ades en la riviere

De cel fleuve qui Nil a non [...] (Le best. divin, 224).

Le cocodrille c'est uns serpenz sauvages cui li comuns de la gent apelent caucatrix. Sa nature si est que quant il troeve i home, si le deveure, et quant il l'a dèvoré si le pleure toz les jors de sa vie (Best. d'amour, 35).

[...] vous $m^{\prime}$ avez fait mention en vostre requeste $d^{\prime} u n$ chocatrix, qui est apelez par son droit non cocodrilles (Rèp. della Dame, 88).

De las dos últimas citas deducimos que cocatriz sería el nombre con el que "la gente común" aludía al cocodrilo. Las dos voces, la culta y la "común" no se excluyen necesariamente, y con frecuencia conviven o se alternan en una misma obra, dando pie a interesantes comentarios metalingüísticos. Fresne Du CANGE, Glossarium (1937, t. II), remite a un texto de Jacques de Vitry escrito en torno a 1240:

In hoc autem flumine vidimus quaedam monstra, quae cocodrilli nuncupantur, gallice autem caucatrices, quae hominibus et equis insidiantes, quicquid dentibus attingunt, devorant (VITRY, 1717, 268).

Por otra parte el mismo Tresor de Latini según la edición Carmody así como la tradución al castellano, pueden servir también como testimonio de este significado.

Dou cocodril c'on apele caucatrix

Or avient que quant i oisel ki a non strophilos i vieut avoir charoigne por mangier, il se boute a la bouche dou cocodril, el li grate tout belement, tant $k^{\prime} i l$ ovre tote la gorge, por le delit du grater; lors vient $i$ autre poisson ki a non idre, et li entre dedens le cors, et $s^{\prime}$ en ist d'autre part, brisant et desrompant son coste, en tel maniere k'il l'ocist. Neis le delfin meismes [...] Et sachiés que cocatrix, ja soit ce k'il nest en euue et vit dedens le Nil, il n'est mie poissons, ains est serpens d'euue; car il ocist home qui il peut ferir, se fiens de buef ne le garist (LATINI, 1948, I, V, 131). 
No aparece ya la identificación explícita de idre con cocatrix. Tampoco se encuentra entre las variantes citadas. El texto, dentro de la confusión que lo caracteriza, induce a creer que cocatriz se refiera al cocodrilo, como se desprende de su título.

En la versión castellana ${ }^{23}$ :

Del Coral

Coral es un animal de quatro pies [...] armado de grandes dientes et de grandes uñas [...] Et acaesçe que quando una ave que ha nonbre estrofiros quiere aver carne para comer, enpuxa un poco la cocatriz et rascala muy quedo fasta que abre la boca con el deleyte del rascar. Et entonçe viene otro pescado que a nonbre ydra et entra por la garganta de la cocatriz et sale de la otra parte, en guisa quel quebranta los costados et mátala. Et otrosy faze el delfin [...] quando vee la cocatriz, entral so el vientre et fierela, de guisa que la mata. Et sabet que la cocatriz, mager que nasçe en el agua [...] non es pescado, ante es serpiente muy mala, ca mata los omes que puede ferir, si con estiercol de buey non guaresçe. Et en aquella tierra ay onbres pequeños, mas son muy ardides en guisa que se ossan parar contra el cocatriz (LATINI, 1989).

El francés cocodril, que aquí se traduce por coral, se identifica con cocatriz, sea al describir su relación con el trochilo (strofiros) y con el delfín, su otro enemigo mortal ${ }^{24}$, sea en la última parte que se refiere a la captura del cocodrilo.

Paralelamente al Tresor, encontramos la voz en la General Estoria de Alfonso el Sabio: "De la natura dela cocadriz, e dela enemiztad del hicneomon con ella", que glosa el pasaje citado de Plinio, relativo al cocodrilo:

En el Nilo á una bestia que llaman cocadriz [...] quando la cocadriz esta en la ribera e se quiere adormir, uiene aella una aueziella que dizen rey passaro, e posal en la boca, et pica le las migaias quel falla aderredor della quel fincan y del pescado que come [...] El hicneomon [sic] coges a andar por la ribera e anda buscando la cocadriz pora lidiar con ella como con su enemigo naturalmientre, mas non se enloda como quando ua lidiar con las

23 En la primera edición del bestiario de Baldwin (LATINI, 1982), el capítulo correspondiente, allí el 125, llevaba el título: Del Coral e de la Cocatriz que estaba en contraste con la identificación de los dos animales dentro del texto y que en la nueva edición ha modificado.

24 Véase PLINIUs (1986), I. VIII, cap. 38; cfr. IsIDORO (1911), XII, 6, 11. 
serpientes; et pues que uee a la cocadriz assi estar durmiendo boca abierta, uiene pora ella manso e muy quedo, e alança se de luenne e dal salto dentro enla garganta [...] E desta guisa es ell hiçneomon enemigo dela cocadriz en la tierra (AlFonso X el SABIO, 1930, vol. I, 222a).

Aquí la General Estoria se aparta voluntariamente del texto de Plinio traduciendo crocodilus por "una bestia que llaman cocadriz"; en cambio, denomina hicneomon a su enemigo, aludiendo a su etimología cuando dice "e anda buscando la cocatriz". No obstante, la alternancia de esta voz con la derivada de en(h)ydros continúa siglos después; así, en 1490, en el Universal Vocabulario de Palencia, s.v. ydra: "[...] idra viene de ydor que es agua, e idras es un animal que salta en la boca del cocodrilo y lo despedaça".

Recapitulando sobre los testimonios que hemos citado hasta ahora relativos al Tresor advertimos que la identificación de ydre con cocatriz se encontraría en una parte de los manuscritos entre los que se encuentran los siete que han determinado la corrección en la edición de Chabaille y los que sirvieron de base a las vulgarizaciones de Giamboni y Copons. En cambio cocatriz, 'cocodrilo', se encuentra en otra rama de testimonios que Carmody ha tenido en cuenta para su edición, así como aquéllos en los que se basa el editor de la traducción del texto al castellano.

Fournival y Alfonso el Sabio, contemporáneos de Latini, emplean la voz para denominar al cocodrilo. Es importante recordar que Latini estuvo en España en 1260, en la corte de Alfonso X, con la misión diplomática de conseguir el apoyo del monarca a la causa de los güelfos, y a su retorno a Italia se vio obligado a huir y refugiarse en Francia (Arras, París y en Bar-sur-l'Aube), donde escribió el Tresor. Era la época en que estaba en auge la "escuela" de Orleans, de la que surgió la Biblionomia de Fournival. Es extraño que Latini, sin que haya antecedentes, al menos documentados ${ }^{25}$, llamara cocatriz a la ydre en un texto donde se habla del cocodrilo, y cuando "la gente común lo llamaba cocatriz". Por otra parte en Tresor I, IV, cap. 123, leemos: "Puis vient la terre de Perse, entre Inde et la mer Rouge [...] puis i a iij isles en quoi naissent li quocatrix, qui ont XX piés de lonc" (también en la edición de Chabaille). Las dimensiones se corresponden con las de los cocodrilos, como confirman los autores y el mismo Latini en el capítulo relativo, y no tanto con las de una hydrus.

No se puede hipotetizar en el texto una confusión de ydre con el basilisco, el cual también fue llamado cocatriz ${ }^{26}$, porque su pequeñísimo tamaño

25 Véase Frédéric Godefroy (1938), vol. 2. Además del de LatinI, cita del Lapidario de Berne, 363 (Pannier): "Li cocatrix et li griffon".

26 Para los significados de la voz en francés, algunos de ellos esporádicos, vid. W. VON WARTBURG (1928), FEW, t. II, 66b, s.v. calcare. En ámbito inglés, la identificación con el basilisco aparece ya en la versión de la Biblia llevada a cabo por John Wycleffe en 1382 y posteriormente se recoge en la Biblia de 1611, autorizada por Jacobo I (en Isaías 11:8; 14:29: 59:5; Jeremías 8:17). Vid. P. A. RoBIN (1932), 83-95. 
está ya testimoniado desde Plinio ${ }^{27}$, y aparece una amplia descripción en Tresor (I. I, IV, cap. 141), sin que allí se mencione cocatriz.

Por lo pronto nos limitamos a dejar constancia de la dificultad de interpretación de la voz en el texto de Latini, esperando que los especialistas del Tresor nos ayuden a echar más luz sobre este capítulo.

En la edición de los Bestiarios catalanes de S. Panunzio (1988), lo calcatrix, masculino y femenino, y las variantes calchatrice y serp caltri, denominan también al cocodrilo:

De la natura del Calcatrix e de la sua significació:

Lo calcatrix si és una manera de serpent qui és fort gran e grossa, e la sua color és quaix roya. E ha aytal natura: que si ella troba algun home, que.l se menge, e puys, com l'á menjat, ella.l plora tots temps de sa vida.

Un testimonio que documenta el uso en catalán y el inicio de la decadencia del vocablo se encuentra en el libro de Bernat Fenollar (s. XV-XVI), Regles d'esquivar vocables (FENOLLAR, 1950, 137-152), donde recomienda: "Evitar de dir [...] calcatrix per dir cocodrilo".

La voz tiene asimismo una larga presencia en los diccionarios españoles, desde el Diccionario de NeBRIJA (1951, 101), hasta, extrañamente, el Diccionario de María Moliner, que lo recoge, aunque como anticuado (MoLINER 1980, s.v. coca: "cocadriz (ant., fem.) cocodrilo"), por seguir el DRAE ${ }^{28}$, donde viene emparejado con cocodrilo.

En el Diccionario de Nebrija, s.v. crocodilus: "animal est quadrupes, quod in terra [et] aqua vivit. La Cocatriz, es un animal de quatro pies, á modo de lagarto particular de las tierras regadas del Nilo. Ay dos suertes de él".

Las dos especies de cocodrilo al que se refiere Nebrija serían el "cocodrilo de tierra", varanus griseus o arenarios, y el cocodrilo de agua, crocodilus niloticus. Esta distinción la encontramos ya en Plinio (PLINIUS, 1986, I. XXVIII, cap. 119) y en T. de Gaza (véase HAUPT, 1869, vol. 3, cap. 41).

El Diccionario de Autoridades (s.v. cocatriz) remite a Nebrija e identifica cocatriz y cocodrilo: "Serpiente que se cría en el Nilo, lo mismo que crocodilo".

Acerca de su etimología los estudiosos se hallan divididos en dos grupos: los que sostienen que la voz proviene del latín calcare: "pisar", referido al ichneumon que destruía los huevos del cocodrilo, y los que la consideran procedente del griego krokodeilos, lat. crocodilus, el cual a través del lat. medieval cocodrillus se convirtió después en cocatrix. dine".

27 PLINIUS (1986), I. VIII, cap. 33: "duodecim non amplius digitorum magnitu-

28 Real Academia Española (1970), DRAE, s.v. cocadriz: "Del b.l. cocatrix-icis, y éste del lat. crocodilus". Y s.v. cocotriz: "De cocadriz", ambos con el significado de "cocodrilo". 
Entre los primeros se encuentran J. A. Simpson y E.S.C. Weiner (OED, 1989, vol. 3, 412), así como J. Corominas y J. A. Pascual, los cuales explican el castellano cocatriz: 'cocodrilo' como resultado de la confusión entre la mangosta y el cocodrilo: "En bajo latín cocatrix, debido a influjo del b. lat. calcatrix (derivado de calcare 'pisar') 'mangosta', animal que los egipcios adoraban por destruir los huevos de cocodrilo, de donde la confusión medieval entre los dos animales" (DCECH, 1980-1991, vol. II, s.v. cocodrilo). Esta explicación no tiene en cuenta los pasos previos de ichneumon a en(h)ydros e ydrus, antes de que se confundiera con calcatrix. La etimología (de calcare: 'pisar'), que podría ser válida si se piensa en la mangosta, no lo es tanto para ydrus 'serpiente de agua' ${ }^{\prime 2}$.

La tesis del DCECH se apoya en W. von Wartburg, FEW, que sostiene la confusión entre el término calcatrix, referido al ichneumón, con cocodrilo, propiamente dicho, debido a la semejanza fonética del nombre y a que se trataba de un animal extraño, desconocido en Europa. La voz, según Wartburg, habría entrado en el español con el significado de cocodrilo, a través de los bestiarios franceses.

En cuanto al significado que da a la voz, primero 'mangosta' y posteriormente, por confusión, 'cocodrilo', Wartburg se basaba, a su vez, en los diccionarios de F. Godefroy y TOBLER-LOMMATZSCH (1956, vol.2, 512), que aportaban como testimonio las citas del Tresor de Latini, en la edición de Chabaille (sin conocer la variante cocatriz: 'cocodrilo' testimoniada en las nuevas ediciones) y que dejaban sin comentar el hecho que apareciera ydre en los textos citados.

A partir de nuestra investigación nos inclinamos a favor de la segunda teoría etimológica, sostenida por W. W. SKEAT (1879) y P. A. ROBIN (1932, 90-91) que postulan que se trata de una forma abreviada de "cocodrillus", de origen francés. Los primeros textos conocidos que documentan el término: Bestiaire divin (1210), J. Vitry (1240) y Bestiaire d'amour (1250), la usan para denominar al cocodrilo. Sólo posteriormente, durante la segunda mitad del XIII y en el XIV, algunos autores, inciertos acerca de su naturaleza, la usaron para denominar a otros animales diversos del cocodrilo, como la hydrus y el basilisco.

En España la voz se usa siempre referida al cocodrilo, excepto en la traducción catalana del Tresor.

De los textos literarios españoles que cita DCECH: coquedriz en D. Juan Manuel, cacotriz en Gómez Manrique (DHist), y cocadriz en Gen. Est., la más antigua y que ofrece una explicación más detallada, es ésta última (vid. supra).

29 Cfr. DECLC, s.v. cocodril. Aquí el autor es más cauto en cuanto a la etimología: "encara que aquell [calcatrix] es presenta com si fos el derivat de calcare 'petjar'". Cfr. DCVB, s.v. calcatrix. 
La variante coquedriz, de D. Juan Manuel se encuentra en el Libro del caballero et del escudero ${ }^{30}$, cap. 40 "De cómo el caballero anciano responde al caballero novel qué cosa son las bestias":

[...] Otras bestias ha y que se crian á las veces en el agua et á las veces en la tierra, así como coquedrices, et los castores et sos semejantes.

Ciceron en De natura deorum, I, 37, 103, enumera también entre los animales que viven en agua y en tierra ("animalia incipites") el castor y el cocodrilo y esta clasificación se encuentra ya en Aristóteles ${ }^{31}$.

El DHLE (II, 467) cuando remite al Cancionero de G. Manrique, se refiere a las Coplas contra los pecados mortales, compuestas en parte por Manrique (Foulché-DelbosC, 1912, vol. I, 129). Allí, donde habla la razón contra la avaricia, se encuentra la siguiente comparación:

Cocatriz es sola una / animalia que te toca, que tiene grande la boca / y salida no ninguna;

y por la vista de alguna / me fundo por esperencia, y digo que es la dolencia / tuya y la desta comuna.

De esta creencia respecto al cocodrilo da testimonio Piero Valeriano en I leroglifici:

Altri dicono, ch'è per il cocodrillo, la crapula significata, percioch'egli non hà il meato per donde si sogliono gli escrementi mandar fuori, la onde è necessario, che per la bocca ogni cosa habbila digestita, ò non digestita, vomiti, e ributti (Valeriano BolzanI, 1626, I. XXIX, 369).

Y Hugo de S. Victor, De Bestiis, en el capítulo relativo al cocodrilo (VICTOR, 1879, I. II, cap. VIII), lo relaciona con los avaros: "Cujus figuram portant hypocritae sive luxuriosi atque avari" 32 .

En vista del interés de la voz nos ha parecido útil agregar algunos testimonios a los citados por Corominas.

30 Véase Pascual de Gayangos (1860), t. LI, 249.

31 ARISTÓteles (1927), I. I, 1, 6: "quaedam vero in humore quidem degunt atque inde victum quaerunt, verum aerem recipiunt (spirando,) non aquam, et extra eam pariunt: cujusmodi multa sunt, cum pedestria, ut lutra (enydris), latax, crocodilus ..., tum carentia pedibus, ut hydrus".

32 Prudencio (1948), 70, vv. 584-588, llama calcatrix mundi a la Avaricia, pero allí calcatrix no hace referencia a un animal. 
Encontramos cocadrid en General Estoria, parte IV, en el libro relativo a Alejandro Magno: "De como vino el rey Alexandre a una tierra et fallóla toda poblada de mugieres et ningun varon" (Alfonso X El SABIO, 1990, 104): "Et es el hipopotamo caballo de la mar, et habie los pechos cuemo cocadrid..." Así como en el Libro del grant acedrex e del acedrex de las diez casas:

E la otra [pieza] que está dell otro cabo del Rey a la mano derecha es a semejança de la Cocatriz que es bestia e pescado, y está fecha como lagarto y cría en las aguas dulçes e señaladamientre en el grant río que llaman Nilo; e ha tan grant fuerça que, teniendo los dos pies de çaga o la cola en el agua, no ha cosa que tome en seco que non tire a ssí por fuerte que sea. E quando quiere tomar alguna cosa faze semejança que cata a otra parte por segurarlo e depués torna assoora en sosquino e va tras ello fasta que lo toma: e a essa semejança la fizieron que jogasse en este acedrex, ca anda en sosquino por todo el tablero o a la primera casa o a quantas quisiere (AlfONSO X el SABIO, 1996, 124).

Y más adelante:

E queremos vos mostrar quál es la mejoria que han los unos juegos sobre los otros, porque el que con ellos jogare que los coñosca, que no dé el mejor juego por el raffaz. El Rey es mayor y mejor juego segunt desuso dixiemos e só el Rey, la Aanca es mejor juego que el Unicornio [...] e el León mejor que la Cocatriz e la Cocatrix es mejor que la Zaraffa [...](ALfonso X el SABIO, 1996, 126).

Para jugar al "Grant Acedrex" se pueden usar también unos dados de ocho caras que valen de 8 a 1 punto: "E porque el rey es mayor e mejor ha los ocho puntos [...] E la cocatriz los tres" (Alfonso X El SABIO, 1996, 128130).

El manuscrito j. T. 6, de fines del s. XIII, conservado en la Real Biblioteca del Escorial, está ilustrado con miniaturas. Una de ellas (fol. $82 v$ ) representa a dos hombres de alto rango social que juegan al "Grant acedrex". Sobre el tablero se pueden ver las piezas colocadas en posición inicial. Entre éstas se halla la cocatriz que tiene la forma de un cocodrilo.

Un testimonio muy interesante lo constituye Andanças e viajes de un hidalgo español de Pero Tafur, escrito en torno al año 1454 y en el que relata los viajes que realizó entre 1436 y 1439. Allí alternan cocatriz y la forma haplológica cotriz, con una amplia descripción del animal:

Ay en esta rivéra [del río Nilo] unas bestias que se crian dentro del agua, que llaman cocatriz [...] Estas bestias suelen salir fuera del agua çinco ó seys pasos, é quando faze sol están mucho adormeçidas é los que las van a matar, llevan un asta de lança é en cabo un rallon con orejas, que, quan- 
do entra, aprieta, é al tirar afierra en la carne ${ }^{33}[\ldots]$ Ésta es en todo fechura de lagarto; tienen los dientes macho é fembra arriba é abaxo, é por esto dizen que, quando travan de alguna cosa, non pueden soltar tan ayna. Éstas fuyen en la tierra de qualquier cosa, porque aquella les es estraña. Déstas vi muchas yo por esta rivera (TAFUR, 1982, 74-75).

El término cotriz (probablemente una haplografía del copista) aparece al describir la gerba, una barca particular usada para transportar viajeros y mercancías por el Nilo:

[las gerbas] llevan contínuamente tres atabales, uno á popa é otro á proa é otro á medianía, por despantar las cotrizes que dixe (TAFUR, 1982, 76).

En otros pasajes de la obra refiere que el sultán de Egipto enviaba, como presentes, a reyes de otros países las pieles de la cocatriz:

[el sultán de Egipto] embióle á rogar [al Adelantado de Damieta] que, si tenía algunt cuero de cocatriz que embiase al rey de Chypre, que le avíe a rogar; é ofreçiose que avían muerto una, é estava fresca é olíe muy mal, tanto que mejor fuera aver traído una fija del Adelantado muy fermosa qué él allí teníe, que'l cuero de la cocatriz (TAFUR, 1982, 119).

Y también cómo éstas pieles podían verse expuestas en los portales exteriores del Palacio de la Señoría (hoy Ducal) de Venecia:

[...] é en aquellos portales los extranjeros ponen sus armas; é áun allí están çiertos cueros de aquellas bestias que dizen cocatrizes, quel soldan de Babylonia, por cosa mostruosa, embió presentados á la Señoría (TAFUR, 1982, 207-208).

Respecto a las dos últimas citas, es interesante señalar asimismo algunos textos franceses citados por V. GAY (1887, s.v. cocodrilo), que aluden a la costumbre de exhibir sus pieles en plazas e iglesias, colgados del muro, y a la creencia de que éstas alejaban el peligro de la peste ${ }^{34}$.

33 De paso señalamos que el término asta de lança es empleado por JUAN RUIZ en sentido figurado en $\angle b a$ 1602ab: "De todos buenos deseos e de todo bien obrar / fagamos asta de lança, e non queramos cansar / con fierro de buenas obras los pecados amatar: / con estas armas lidiando, podémoslos amansar".

MARCO POLO (1982), cap. CIII, refiere un modo semejante para capturar "il gran serpente" o cocodrilo: "E [li cacciatori] hanno un palo di legno grosso e forte, e in quel palo è fitto un ferro d'acciaio fatto com'un rasoio [...]".

34 Las citas que aporta son de: Journal d'un bourgeois de Paris, 49; J. Belon, Observations, 1.2, cap. 32; Secrets d'Alexis, part. 2, 1.3, 37. 
Hemos dejado para el final la enmienda cocatriz que Corominas propone para el verso 881c del Lba.

Del análisis de todos los textos en lengua castellana que hemos citado se confirma que la voz cocatriz, así como sus variantes, denomina al cocodrilo, sin que hayamos podido encontrar testimonios de significados diversos. El significado que Corominas da a la voz cocatriz: 'cocodrilo', sería pues el más apropiado en un texto castellano medieval. Queda por resolver si se acepta la enmienda para los versos 881 a-d:

si no parlás la picaça más que la codorniz non la colgarien en plaça nin reirien de lo que diz: castigadvos, ya amiga, de otra tal cocatriz, que todos los omnes fazen como don Melón Ortiz.

La explicación de Corominas es la siguiente: "Se trata del supuesto arrepentimiento tardío del cocodrilo después de sus fechorías devoradoras, revelado por las lágrimas que le provoca la digestión". Según esta interpretación la metafora aludiría indirectamente a la picaça y directamente a doña Endrina, ya que ambas se lamentan con arrepentimiento tardío.

J. JURADO (1968), disociándose de esta interpretación, sostiene que cocatriz se refiere sólo a la picaça, y con ella se alude a la leyenda de que el cocodrilo muere "por permanecer incautamente con la boca abierta", lo mismo que la picaça muere "por haber abierto el pico". Ha de tenerse en cuenta, sin embargo, que durante toda la Edad Media se creyó que el cocodrilo carecía de lengua por estar ésta pegada casi completamente a la mandíbula inferior, y que por su "silencio" se creía que el cocodrilo era símbolo de la divinidad (Vid. Valeriano, 1626, 366 y ss.).

Existe la posibilidad, hasta ahora no considerada, que cocatriz, entendido en sentido metafórico, vaya referido a don Melón que, como el cocodrilo "tiene un fingido llanto, con que engaña a los passageros, que piensan ser persona humana, afligida y puesta en necessidad, quando vee que llegan cerca dél, los acomete y mata en la tierra" ${ }^{35}$. Don Melón atrae a doña Endrina, con la ayuda de Trotaconventos, mediante engaños y falsas promesas y después de que haya aceptado verse con él en casa de la medianera la toma por la fuerza, a traición. Este significado de cocodrilo, "traidor", lo podemos encontrar también en otros textos ${ }^{36}$. En cuanto al género, cocatriz es ambivalente, puede referirse a sustantivos masculinos o femeninos. Por otra parte los versos $c$ y d guardan estrecha relación, el último está subordinado al anterior con un que ilativo, y si cocatriz se refiriera a la picaza se vaciaría de sentido.

35 S. de Covarrubias (1953), 330. Cfr. Aut., 392. V.q. Alfonso X el Sabio (1996).

36 LOPE DE VEGA (1983), La Filomena, vv. 428-429. Para otros textos que se refieren a la naturaleza traicionera del cocodrilo, véase J. JURADO (1988), n. 39. 
Siguiendo nuestra hipótesis, los dos últimos versos de la estrofa constituirían una advertencia en boca de Trotaconventos (¿o del Arcipreste?) a no caer de nuevo en el mismo error: ¡Prevenios ya amiga de otro "cocodrilo" tal, porque todos los hombres hacen como D. Melón!, acorde con las advertencias que da el Arcipreste en 905ab: "La que por ventura es o fue engañada, / guárdese que non torne al mal otra vegada"; en 909a-d: "Entiende bien la estoria de la fija del endrino: / díxel' por te dar ensienplo, mas non porque a mi vino; / guárdate de falsa vieja, de riso de mal vecino, / sola con omne no $t^{\prime}$ fies nin te llegues al espino".

Esta interpretación substancialmente es acorde con la que proponen $\mathrm{M}$. Morreale (1968): "prepárate para otra ocasión semejante" y G. CHIARINI: "un altra volta, in circostanze analoghe seguite l'opposta linea di condotta" (JUAN Ruiz, 1964, 165, en n.).

El contexto donde se incluyen los versos es una exhortación a la prudencia y al silencio para encubrir el mal que ha sufrido doña Endrina, es decir, la pérdida del honor. También en el Rèponse de la Dame al Bestiaire d'Amour de Richard de Fournival citando la cocatris, la amada dice que la teme (aludiendo metafóricamente a un hombre), y que si fuese engañada por una que obtuviera de ella lo que desea, gozaría de poca estima y sería despreciada por quien ahora la respeta; por ello su comportamiento sería el de ocultarlo mientras pudiera (FouRNIVAL, 1978, 81 y ss).

Un documento que podría avalar la enmienda de Corominas y esclarecer el significado de cocatriz en el contexto, es una esparsa del s. $\mathrm{XV}^{37}$, analizada por K. Whinnom (1977), que evoca el episodio de doña Endrina y don Melón:

Sepa el rey y sepan todos
quantos en su carta son
que por muy ceuiles modos
doña Endrina y Don Melón
se casaran hasta los codos.
Don Melón es desaguisando
a guisa de cocatriz
doña Endrina y su nariz
an de andar siempre picando
entrel cuero y la ceruiz.

JURADO (1988) interpreta desaguisando, o bien desguisando, según la enmienda que defiende, como 'haciendo la digestión', lo que lo lleva a dedu-

37 Se trata de una poesía festiva existente en el ms. 617, fol. $208 v$ de la Biblioteca de Palacio de Madrid, y en una copia de éste, ms. 506, fol. 392v, de la Biblioteca Pública de Toledo, que se refiere a las bodas del duque de Villahermosa con "María de Soto". 
cir que don Melón está en la cama "sesteante", y que doña Endrina, como el trochilo que se posa en la boca del cocodrilo para comer las migajas de pescado que encuentra alrededor, provocándole gran placer, está cosquilleando con sus besos en el cuello a don Melón.

Esta interpretación "maliciosa" no puede ser descartada, si bien llevaría a suponer que los oyentes conocen, no sólo el significado metafórico de cocatriz / cocodrilo, sino también otros particulares más eruditos. Creemos, en cambio, que dado el carácter festivo y popular del poema, el "doble" significado debería ser más directo, en consonancia con la finalidad de este tipo de poesías y con el público al que iban destinadas ${ }^{38}$.

Una interpretación más "popular" sería que don Melón haciendo "desaguisados" (agravios, denuestos), es a la manera de la cocatriz, o sea falso y traidor, y que doña Endrina con su "olfato" o "agudeza" ha de saber buscar su punto débil "entre el cuero y la cerviz". Podrían caber interpretaciones superpuestas, ya que cuero posee un segundo significado alusivo a los odres que contienen el vino, y por analogía al borracho ${ }^{39}$, pero no sabemos si el duque de Villahermosa al que se refiere el poema era aficionado al vino; por otra parte "ser uno de dura cerviz" significa metafóricamente "no tener condición tratable, ni que le puedan domeñar, amigo de su opinión y parecer"40, y esta definición sí cuadra bastante al personaje (vid. WHINNOM, 1977).

De la esparsa nos interesa destacar sobre todo el hecho de que el autor anónimo del siglo XV alude al episodio de don Melón y doña Endrina, identificando a aquél con la cocatriz, como nosotros proponemos para $L b a$, y no se puede descartar, como hipotiza Jurado, que existiera un manuscrito hoy desaparecido donde la lección de 881c fuera cocatriz.

Cabe aún considerar una segunda interpretación, que nos ha sido sugerida por la profesora Margherita Morreale, y es que el Arcipreste con cocatriz haga alusión al juego, a la pieza del mismo nombre que se describe en el Libro del gran acedrex (Alfonso X, 1996, 124). La Cocatriz del "Ajedrez de las doce casas" se mueve por el tablero en diagonal y ataca de través, a traición, a la manera del cocodrilo, con cuya imagen se representa; su juego, respecto al

38 Señalamos aquí la tradición literaria que hace de Don Melón el prototipo de los que se casan. Quevedo en el poema satírico "Boda y acompañamiento del campo", vv. 57-60 (F. DE QUeVEDo, 1964, poema 683): "Don Melón, que es el retrato / de todos los que se casan: / Dios te la depare buena, / que la vista al gusto engaña". Otro poemilla satírico es de Julio Monreal, recogido por ANICETo PAGÉs (1902) s.v. cocodrilo: "[...] un dogal con carlanca en la cerviz; / un baño en cueros vivos de alquitrán / [...] / Estar de un cocodrilo en mancomún, / y vivir cual vivió San Sebastián, / ese es el matrimonio y más aún".

39 Se pueden encontrar numerosos ejemplos en los poemas satíricos de QUEVEDO (1964), entre ellos los números 622, 625, 666 y 725.

40 Vid. CovarRubias (1953), s.v. cerviz; cfr. DRAE, s.v. cuero y cerviz. 
de otras piezas, es calificado de raffaz (cfr. Lba 861c "jugaremos a la pella e a otros juegos raezes"). La voz, entendida como "un mal juego" o "juego peligroso", se refiere a la trampa tendida por don Melón. El verso así interpretado es afín al 753d, por estructura y por contexto: "guardatvos, Doña Endrina, d'estas peranças malas", donde Trotaconventos previene a doña Endrina contra los que pueden tenderle trampas, hacerle un mal juego, (754b mal trebejo). El verbo jugar aparece con frecuencia en el episodio: $861 \mathrm{~cd}, 863 \mathrm{~d}, 867 \mathrm{~b}$.

Esperamos que nuestra modesta aportación sobre el término cocatriz y sus variantes, pueda servir de ayuda para el estudio del término en ámbito hispánico y francés, y para el análisis de los versos del $L b a$ que hemos citado. Nos hemos atrevido también a hipotetizar una interpretación de éstos, sin confrontarla desde un punto de vista formal con las que nos venían propuestas hasta ahora por los especialistas en $L b a$, que el lector encontrará reunidas y analizadas con detalle en el ensayo de Jurado que hemos tomado como punto de partida.

BiBLIOGRAFíA

Alcover, ANTONI M. y Francesc de BorJa Moll (1988): Diccionari català-valencià-balear (DCVB), Palma de Mallorca, ed. Moll.

Alfonso X el SABIo (1930): General Estoria, ed. Antonio G. Solalinde, Madrid, Centro de Estudios Históricos.

Porrúa.

(1990): Antología, est. prelim. de Margarita Peña, México,

(1996): Il libro dei giochi, Paolo Canettieri, ed., Bolonia, Cosmopoli.

ALONSO de PALENCIA (1967): Universal vocabulario en latín y en romance, Madrid, Comisión Permanente de la Asociación de Academias de la Lengua Española.

Ammiano Marcelo (1963): Rerum gestarum. I, Carolus U. Clark, ed., Berolini, apud Weidmannos.

ARISTÓTELES (1927): De animalibus historiae. VI, en Opera omnia graeca et latina, vol. 3, Parisiis, Firmin-Didot.

Blosius Aemilius Dracontius (1961): De Laudibus Dei, cum recensione Eugenii Toletani, en Monumenta Germaniae Historica (Auctorum Antiquissimorum, XIV), Berolini, Societas Aperiendis Fontibus.

CAMERARIO, JOAQUIMO (1654): Symbolorum et Emblematum ex animalibus quadrupedibus desumtorum centuria altera collecta, Francofurti, Impensis loh. Ammony. 
Ciccuto, Marcello (1992): "Tresor di Brunetto Latini", en Letteratura italiana. Le opere. I. Dalle origini al Cinquecento, Torino, Einaudi.

Clerc de Normandie, Guillaume (1970): Le Bestiaire divin, C. Hippeau, ed., Genève, Slatkine Reprints.

Corominas, JoAn y José ANTONio PAscual (1980): Diccionario crítico etimológico castellano e hispánico (DCECH), Madrid, Gredos.

COROMINAS, JOAN (1990): Diccionari etimològic i complementari de la llengua catalana (DECLC), Barcelona, Curial-La Caixa.

CovarRubias, Sebastí́N de (1953), Tesoro de la lengua castellana o española, Martín de Riquer, ed., Barcelona, Horta.

FENOLLAR, BERNAT (1950): Regles d'esquivar vocables o mots grossers o pagesívols, Antoni M. Badia Margarit, ed., Boletín de la Real Academia de Buenas Letras, XXIII, pp. 137-152.

ForCELlinI, AEGIDIO, ed. (1940): Lexicon totius latinitatis, Patavii, Typis seminarii.

FoulchÉ-Delbosc, RAYMOND, ed. (1912): Cancionero castellano del s. XV, Madrid, NBAE.

FOURNIVAL, RICHARD DE (1978): Le bestiaire d'Amour, Richard de Fournival suivi de la Réponse de la Dame, C. Hippeau, ed., Genève, Slatkine Reprints.

Fresne du Cance, Charles du (1937): Glossarium mediae et infimae latinitatis, Paris, Librairie des Sciences et des Arts.

GAY, VICTOR (1887): Glossaire archèologiche du moyen age et de la renaissance, Paris, Libr. de la Soc. Bibliographique.

GaYANGOS, PASCUAL DE (1860): Escritores en prosa anteriores al siglo XV, Madrid, Rivadeneyra.

GodefroY, FrÉdéRIC (1938): Dictionnaire de l'ancienne langue française et de tous ses dialectes, Paris, Librairie des Sciences et des Arts.

Grzimek, Bernhard (1972): Vita degli Animali, Milano, Bramante.

HAUPT, MORIz (1869): Excerpta ex Timothei Gazaei libris de animalibus, en Hermes, vol. 3, Berlin

HENKEL, A. Y A. SCHÖNE (1967): Emblemata. Handbuch zur Sinnbildkunst des XVI und XVII Jahrhunderts, Stuttgart, Metzler.

Holloway, Julia B. (1986): Brunetto Latini. An Analytic Bibliography, London, Wolfeboro.

IsIDORO, SAN (1911): Etymologiarum sive originum libri XX, en Wallace M. Lindsay, ed., Scriptorum Classicorum Bibliotheca Oxoniensis, t. II, Oxford, The Clarendon Press.

(1982): Etimologías, J. Oroz Reta y M. A. Marcos Casquero, eds., Madrid, Editorial Católica.

JURAdo, José (1988): "Libro de Buen Amor, 881c: La alusión a la 'cocatriz", Boletín de la Real Academia Española, 68, pp. 433-454. 
LATINI, Brunetto (1839): II Tesoro di Brunetto Latini, volgarizzato da Bono Giamboni, pubblicato secondo l'edizione del 1533, Luigi Carrer, ed., Venezia, tip. del Gondoliere.

(1863): Li livres dou Tresor, Polycarpe Chabaille, ed., Paris, Imprimerie Impèriale.

(1871): Il Tesoro de Brunetto Latini, volgarizzato per Bono Giamboni, Luigi Gaiter, ed., Bologna, Romagnoli.

(1948): Li livres dou Tresor, Francis J. Carmody, ed., Berkeley and Los Angeles, University of California Press.

(1976): Llibre del Tresor, C.J. Wittlin, ed., Barcelona, Barcino.

(1982): The Medieval Castilian Bestiary from B. Latini's "Tesoro",

ed. y estudio de Spurgeon Baldwin, Exeter, Exeter University.

(1989): Libro del tesoro. Versión castellana de Li livres dou tresor,

ed. y estudio de Spurgeon Baldwin, Madison, The Hispanic Seminary of Medieval Studies.

LOPE DE VeGA, FÉLIX (1983): La Filomena, en Obras poéticas, José Manuel Blecua, ed., Barcelona, Planeta.

Moliner, María (1980): Diccionario de uso del español, Madrid, Gredos.

Morreale, MarGHerita (1968): "Más apuntes para un comentario literal del

Libro de Buen Amor con otras observaciones al margen de la reciente edición de G. Chiarini", Boletín de la Real Academia Española, 48, pp. 117144.

NebriJA, ANTONIO de (1951): Dictionarium Aelii Antonii Nebrissensis, Martínez de Cala y Jaraba, ed., Madrid.

PAGÉs, ANiCeto (1902): Gran diccionario de la Lengua Española (de Autoridades), Barcelona, Ed. Selección.

Panunzio, Saverio, ed. (1988): Bestiaris, Barcelona, Barcino.

Physiologus (1936): Franciscus Sbordone, ed., Mediolani-Genuae-RomaeNeapoli, "Dante Alighieri".

(1960): Der Fhysiologus, Otto Seel, ed., Zürich und Stuttgart, Artemis Verlag.

(1975): Il Fisiologo, Francesco Zambon, ed., Milano, Adelphi.

Pisides, Georgios (1860): Hexaemeron, en Jacques-Paul Migne, Patrologiae graecae, vol. XCII, Parisiis, J.-P. Migne editorem.

Plinius SeCundus, Calus (1986): Naturalis Historia, Ludovicus lan y Carolus Mayhoff, eds., Stuttgart.

Polo, Marco (1982): Il Libro di Marco Polo detto Milione, Torino, Einaudi.

Prudentius Clemens, Aurelius (1948): Physicomachie, M. Lavarenne, ed., Paris, Les Belles Lettres.

Quevedo, Francisco de (1964): Obras completas. I: Poesía original, José Manuel Blecua, ed., Barcelona, Planeta. 
Real Academia Española (1936): Diccionario Histórico de la Lengua Española (DHLE), Madrid.

(1970): Diccionario de la Lengua Española (DRAE), Madrid, $19^{\underline{a}}$ ed. (1979): Diccionario de Autoridades (Aut.), Madrid, Gredos, ed.

facs.

RoBIN, P.A. (1932): Animal Lore in English Literature, Londres, John Murray.

RuIz, JUAN (1964): Libro de Buen Amor, Giorgio Chiarini, ed., Milano-Napoli, Riccardo Ricciardi.

dos.

(1967): Libro de buen amor, Joan Corominas, ed., Madrid, Gre-

(1974): Libro de Buen Amor, Jacques Joset, ed., Madrid, Espasa

Calpe.

(1988): Libro de Buen Amor, G.M. Gybbon-Monypenny, ed., Madrid, Castalia.

SAINT-DENIS, E. DE (1947): Le vocabulaire des animaux marins en latin classique, Paris, Klinksieck.

Salmasio, Claudio (1689): Plinianae exercitationes in Caji Julii Solini polystor ex veteribus libris emendatus. I, Trayecti ad Rhenum, apud Joh[ann] vande Water.

SkEAT, WALTER W. (1879): An Etymological Dictionary of the English Language, Oxford, Clarendon Press.

SolINUS, C. IUILIUS (1864): Collectanea Rerum Memorabilium, recognovit Th. Mommsen, Berolini, Nicolai.

Stephano, Henrico (1954): Thesaurus Graecae Linguae, Graz, Akademische Druck-U. Verlagsanstalt

StRABO (1852): Geographica, recognovit Augustus Meineke, Lipsiae, Sumptibus et Typis B.G. Teubneri.

TAÜN, PHILIPPE DE (1970): Le Bestiaire, Emmanuel Walberg, ed., Genéve, Slatkine Reprints.

TAFUr, Pero (1982): Andanças e viajes de un hidalgo español, Marcos Jiménez de la Espada, ed., Barcelona, El Albir.

THE BOOK OF BEASTS (1954): The Book of Beasts: being a translation from a Latin bestiary of the twelfth century, ed. T.H. White, Londres, Jonathan Cape.

The OXford ENGlish Dictionary (1989) (OED): Oxford, Clarendon Press, $2^{\mathrm{a}}$ ed.

THesaurus liNGUAe LATINAE (1900): editus auctoritate et consilio Academiarum quinque Germanicarum Berolinensis, Gottingensis, Lipsiensis, Monacensis, Vindobonensis, Leipzig, Teubner.

TOBLER, AdOLF y ERHARD LOMMATZSCH (1956): Altfranzösisches Wörterbuch, Wiesbaden, Weidmannsche-Steiner.

Valeriano Bolzani, Giovanni Pietro (1626): I leroglifici, ovvero commentarii delle occulte significationi de gl'Egittij et altre nationi, Venezia, Gio[vanni] Battista Combi. 
Victor, Hugo de SAN (1879): De Bestiis et aliis Rebus, en Patrologia latina, t. CLXXV, Jacques-Paul Migne, ed., Parisiis, apud Garnier Frates Editores et J.-P. Migne successores.

VITRY, JACQUES DE (1717): Tusculani Historiae Orientalis, en EDMOND MARTÈNE, Thesaurus Novus Anecdotorum, t. III, Paris.

WARTBURG, WALTER VON (1928): Französisches Etymologisches Wörterbuch (FEW), Bonn und Leipzig, Kurt Schroeder Verlag.

Whinnom, Keith (1977): "A Fifteenth-Century Reference to Don Melón and Doña Endrina", Journal of Hispanic Philology, II, pp. 91-101. 